\title{
meeting report
}

\section{Keeping up with calcium}

\section{Conference on calcium-binding proteins and calcium function in health and disease}

\author{
Claude B. Klee \& Anthony R. Means ${ }^{1,+}$
}

Laboratory of Biochemistry, Center for Cancer Research, National Cancer Institute, National Institutes of Health, Building 37, Room 6106C, 37 Convent Dr. MSC 4255, Bethesda, MD 20892-4255 and ${ }^{1}$ Department of Pharmacology and Cancer Biology, Duke University Medical Center, Box 3813, Durham, NC 27710, USA

Received June 3, 2002; revised July 23, 2002; acepted July 26, 2002

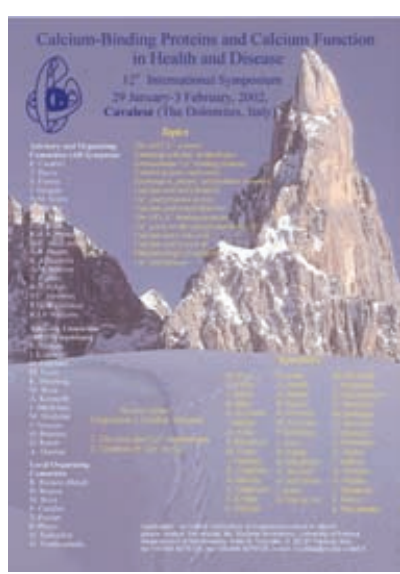

The 12th International Symposium on Calcium-Binding Proteins and Calcium Function in Health and Disease was held in February, 2002, in Cavalese, Italy. The 4-day meeting consisted of nearly 60 plenary presentations and over 180 posters.

\section{Introduction}

Our understanding of how calcium functions as a second messenger continues to increase at an incredible pace. This symposium covered most, if not all, aspects of the many roles of calcium in the regulation of cellular processes and ranged from calcium imaging through to the structure and function of calcium-binding proteins. Emphasis was placed on elucidating the involvement of these proteins in the pathogenesis of a myriad of human diseases using genetic engineering technology. Unfortunately, so many interesting presentations were given that this review of the meeting can only cover a few topics that the authors feel resulted in conceptual advances in this broad field of science. We apologize to our many colleagues whose work is not given the coverage it deserves due to space limitations.

\section{Methodological advances}

It is fascinating to consider that the cell contains $>1000$ signaling proteins and only 10 second messengers. Calcium may be the most pervasive of the latter, as it can be spatially and temporally controlled with remarkable finesse and interacts with a dizzying array of proteins to execute its many regulatory functions. In response to signaling, calcium is transiently released into the cytosol from the external medium or the endoplasmic reticulum lumen and is sensed and modulated by a variety of calciumbinding proteins. Calmodulin, which binds calcium due to its repeated 'EF-hand' motif, is the most prominent member of this class of proteins because of its ability to activate a large number of target proteins, particularly those involved in protein phosphorylation, cyclic nucleotide metabolism and calcium homeostasis. T. Meyer (Stanford, CA) pointed out that analysis of the human genome predicts the presence of a common calciumbinding motif in a number of the proteins encoded. For example, 83 EF-hand proteins that could bind calcium in a manner similar to calmodulin have been detected, and 73 others have been found to contain a C2 domain, a conserved calcium-binding motif originally described in protein kinase C (PKC). Meyer used a method called total internal reflective fluorescence to measure the rate of the movement of cytosolic PKC and found that this enzyme rapidly diffuses through the cell. Upon activation, it 'hits and runs' from the plasma membrane, and it is speculated that this translocation is due to the release of cofactors such as calcium that 'hold' the protein at the site where it can then phosphorylate its target membrane proteins (Teruel and Meyer, 2002). The results of these experiments suggest that the hit-andrun frequency is dependent on calcium influx. This remarkable technique has now been adapted to perform parallel single cell monitoring of 1000-10 000 cells and should be useful in evaluating how so many calcium-binding proteins can function as receptors to provide the exquisite control of calcium-mediated processes. Other powerful biophysical techniques have been used to address the diversity of calcium signaling. A. Konnerth (Munich, Germany) used two-photon imaging to study the calcium signal initiated by neurotrophins, a family of neurotrophic factors that interact with the cell membrane to support

+Corresponding author. Tel: +1 919681 6209; Fax: +1 919681 7767; E-mail: means001@mc.duke.edu 


\section{meeting report}

neuronal survival and differentiation (Kovalchuk et al., 2002). Monitoring of membrane capacitance on a millisecond timescale, combined with the use of caged calcium and site-directed mutagensis, allowed E. Neher (Gottingen, Germany) to analyze the roles of individual proteins in the coupling of calciuminduced fusion of secretory vesicles to the plasma membrane of chromaffin cells. This is the first example of the kinetic dissection of the complex set of events leading to the release of neurotransmitters at the molecular level (Sorensen et al., 2002). A critical and in-depth analysis of the possible applications of these sophisticated and novel techniques to other proteins and cellular processes will undoubtedly lead to a better understanding of the complex interrelationships of various calcium-signaling pathways in a single cell.

\section{Structural diversity}

The structures of calmodulin complexed with a number of its target peptides and proteins have now been solved (Figure 1). These structures were summarized by M. Ikura (Toronto, ON), who pointed out the diversity of conformations that have been described and how each can be unique to the particular activation mechanism characteristic of the target protein. These various activation mechanisms are illustrated by active-site remodeling in the case of the anthrax adenylyl cyclase (Drum et al., 2002), subunit dimerization in the case of potassium channels (Schumacher et al., 2001) and displacement of autoinhibitory domains in the case of several calmodulin-dependent protein kinases and phosphatases (Hoeflich and Ikura, 2002). The understanding of the various structural conformations of these target proteins helps in the appreciation of the remarkable versatility of calmodulin as a calcium receptor. Consideration of the available structures suggests that calmodulin specificity is inherent in the structure of the calmodulin-binding motif of the target protein rather than in calmodulin itself. Another example of the complexity and versatility of calcium regulation was provided by P. Davis (Kingston, ON), who presented an intriguing structural analysis of the calcium-activated proteases, calpains 1 and 2 (Moldoveanu et al., 2002). These enzymes are unusual in that they contain both EF-hand and C2 calciumbinding domains. Calcium binding to both types of motifs is required for activation and autoproteolysis of the enzymes, and therefore both motifs must act in concert to regulate the biological functions of the calpains. Additionally, the structure of phospholamban was presented by J. Krebs (Zurich, Switzerland) and E. Carafoli (Padova, Italy). Phospholamban is an intrinsic membrane protein that modulates the activity of the calcium pump located in the sarcoplasmic reticulum of striated muscles. A comparison of this new structure with that previously determined for the calcium pump provides some insight into how these two proteins may interact and how the phosphorylation of phospholamban may relieve inhibition of the pump.

\section{Functional advances}

Calcineurin, a calmodulin/calcium-dependent serine/threonine phosphatase, controls the entry of the NFAT (nuclear factor of activated $\mathrm{T}$ cells) transcription factor into the nucleus of $\mathrm{T}$ lymphocytes in response to activating signals and also serves as the target for the immunosuppressive agents, cyclosporin A and FK506 (as reviewed by Crabtree and Olson, 2002). It is now appreciated that this general mechanism of transcriptional control occurs in a number of other mammalian cells and, indeed, in other species (Figure 2). Evidence presented at the meeting supports a role for calcineurin-mediated transcriptional activation in cardiac hypertrophy (E. Olson, Dallas, TX), cardiac development in combination with calreticulin (M. Michalak, Edmonton, $\mathrm{AL}$ ), morphogenesis of sensory neurons (G. Crabtree, Stanford, CA) and determination of the Xenopus dorsoventral axis together with the inositol 1,4,5-trisphosphate (IP3) receptor (K. Mikoshiba, Tokyo, Japan; Saneyoshi et al., 2002). In addition, M. Cyert (Stanford, CA) presented evidence for a pathway in budding yeast that is activated in response to environmental stress and is analogous to the calcineurin/NFAT pathway in mammalian cells. Crz1/Tcn1 $\mathrm{p}$ is the yeast NFAT homolog, and calcineurin promotes its nuclear accumulation by both increasing its nuclear import and decreasing its nuclear export (Boustany and Cyert, 2002). In the Drosophila visual pathway, calcium and calmodulin regulate the rhodopsin cycle. C. Montell (Baltimore, MD) showed that this pathway involves a decrease in the activity of rhodopsin kinase together with a calcium/ calmodulin kinase II (CaMKII)-mediated activation of arrestin, which terminates the light response. In addition, calmodulin increases the activity of a rhodopsin phosphatase, RDGC, encoded by the retinal degeneration $C(r d g C)$ locus of Drosophila. Montell found that this phosphatase contains five EF-hand domains and is therefore regulated by calcium but that it is also regulated by relief of autoinhibition in a manner similar to calcineurin. RDGC, homologs of which have been identified previously in mammals (Ramulu et al., 2001), is only the second member of the calcium/calmodulin-dependent protein phosphatase family to be identified (Lee and Montell, 2001). It will be interesting to elucidate other physiologically relevant roles for this new class of calmodulin-regulated enzymes.

It has long been held sacred that calmodulin binding is necessary to activate all calmodulin-dependent enzymes. In the case of the multimeric CaMKII, binding of calcium/calmodulin to adjacent subunits promotes autophosphorylation, which generates autonomous activity. The group of $\mathrm{H}$. Schulman (Stanford, CA) has identified a variation on this theme whereby an increase in calcium in an N-methyl-D-aspartate (NMDA)-dependent neuron results in the association of CaMKII with the NR2B subunit of the NMDA receptor. This interaction is sufficient to generate autonomous activity in the absence of autophosphorylation. Binding of the NR2B subunit to CaMKII is apparently an allosteric mimic of the normal intrasteric binding of the enzyme's autoregulatory domain, apart from the fact that it promotes persistent activation of the CaMKII kinase, even after the dissociation of calcium/calmodulin (Bayer et al., 2001). It was suggested that the amount of CaMKII bound to the NMDA receptor may reflect a history of synapse activity. It will be interesting to determine whether this novel mechanism of inducing autonomous activity is also applicable to the regulation of other calmodulin-dependent enzymes. 


\section{meeting report}

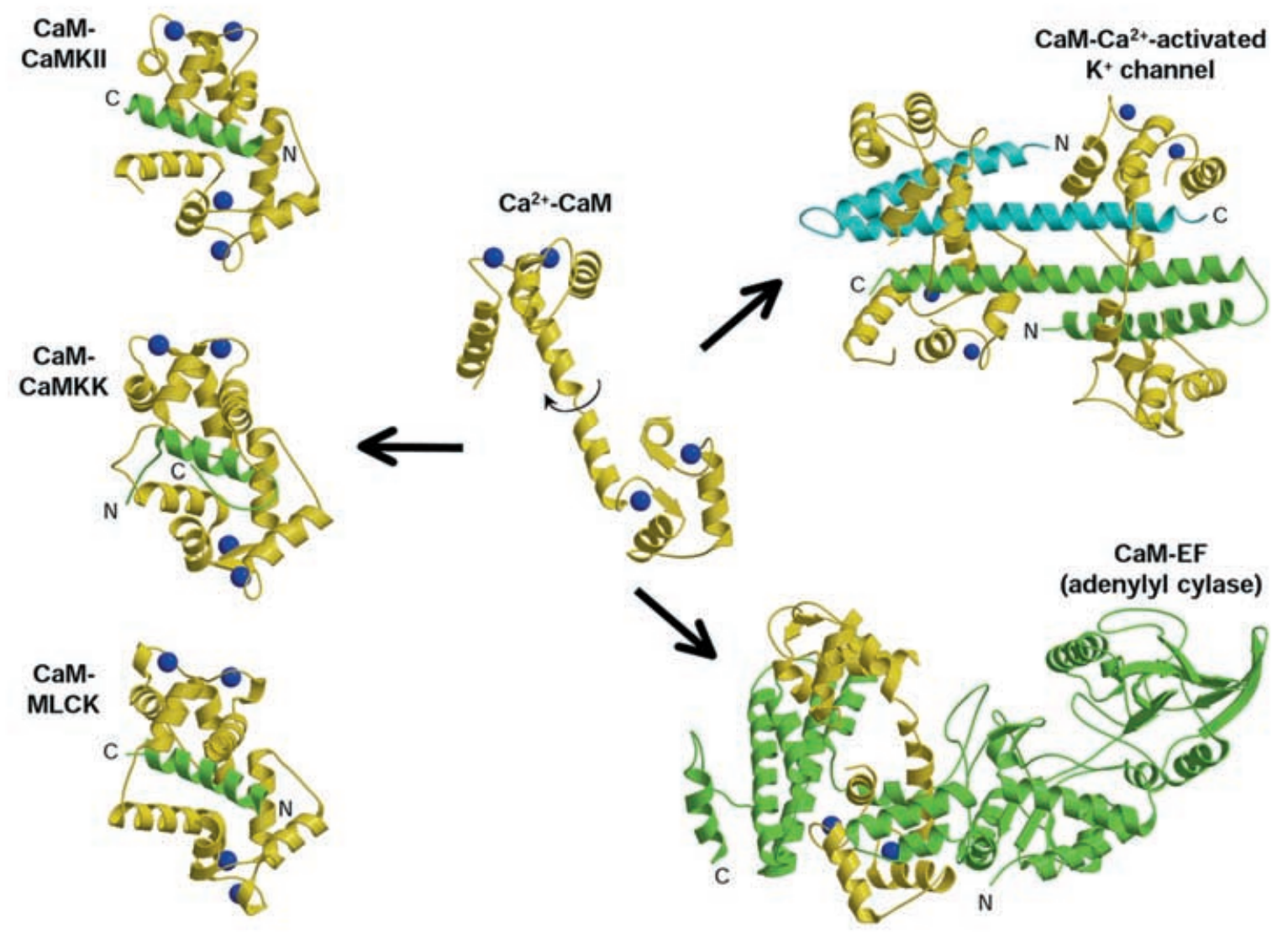

Fig. 1. Comparison of different calmodulin target structures. Centre: calmodulin (CaM) is shown in yellow with its four calcium sites (shown as blue balls) occupied; the arrow indicates the flexibility of the central helix linking the $\mathrm{N}$ - and $\mathrm{C}$-halves of the molecule. Left: the different modes of interaction of CaM (with four calcium sites occupied) with the CaM-binding peptides (shown in green) of CaM kinase II (CaMKII), CaM kinase kinase (CaMKK) and myosin light chain kinase (MLCK). Right: the dimeric structure of the CaM-binding domain of the potassium channels (shown in green and aqua) each bound to a molecule of CaM, with the two N-terminal sites occupied; the structure of Bacillus anthracis edema factor (EF) illustrates how CaM, with calcium bound to its two C-terminal sites, can activate the enzyme by remodeling its catalytic site. (Reproduced with permission from Hoeflich and Ikura, 2002.)

\section{Understanding calcium homeostatic mechanisms}

It has long been appreciated that the mitochondria can accumulate large amounts of calcium, but their role in regulating intracellular calcium fell out of favor 20 years ago due to the low levels found in mitochondria under most physiological conditions (Carafoli, 2002). However, this meeting restored this important organelle to its rightful place in the field of calcium research. R. Rizzuto (Ferrara, Italy) revealed that calcium uptake into mitochondria is a result of histamine stimulation (Vandecasteele et al., 2001). Calcium enters the mitochondrion via a uniporter and, because of the close proximity between these organelles and the endoplasmic reticulum, mitochondrial calcium rises from $\sim 0.2$ to $10 \mu \mathrm{M}$. There seem to be several reasons why this uptake system might be physiologically significant. First, calcium regulates at least three key Krebs Cycle enzymes that are required for the generation of ATP. Secondly, this storage in mitochondria could help to regulate global calcium signals in a spatially and temporally important manner. Finally, excess calcium can cause mitochondria to swell, thereby initiating apoptosis. These important roles for mitochondrial calcium uptake were originally identified in HeLa cells but have also now been shown to be important in mammalian liver cells by A. Thomas (Newark, NJ). In addition it was suggested that, in muscle cells, the mitochondria may be coupled to ryanodine receptors, the highly active calcium channels of the sarcoplasmic reticulum. P. Duchen (London, UK) used photoactivation to generate superoxide from mitochondria-targeted agents such as tetramethyl-rhodamine dyes and observed a release of calcium from the endoplasmic or sarcoplasmic reticulum in response to this artificial oxidative stress. He discussed the idea that this local increase of calcium could result in mitochondrial overload, a further increase in superoxide production, mitochondrial depolarization and eventually a collapse of the mitochondrial membrane potential leading to necrotic cell death (Schuchmann et al., 2001). Indeed, these stress-related events may reflect a response to calcium released from the endoplasmic or sarcoplasmic reticular systems that results in ATP depletion and the initiation of apoptosis. These reactive oxygen species may also play a role in the reversible calcium/calmodulin-dependent inactivation of calcineurin, which provides a mechanism for coupling calcium signaling to the redox potential of the cell, as reported by C. Klee (Bethesda, MD). M. Berridge (Cambridge, UK) also emphasized 


\section{meeting report}

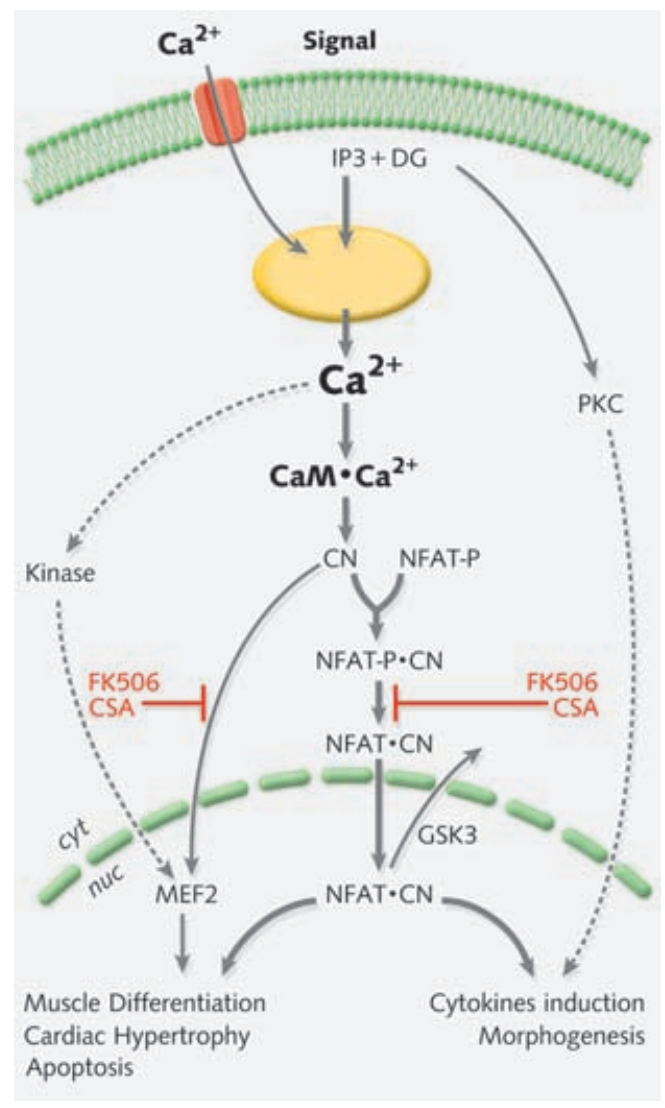

Fig. 2. Schematic representation of calcineurin regulation of gene expression in mammalian cells. Activation of phospholipase $\mathrm{C}$ in response to an external calcium mobilizing signal generates 1,4,5-trisphosphate (IP3) and diacylglycerol (DG). IP3 induces calcium release from internal stores (shown in yellow). Calcium binds to calmodulin ( $\mathrm{CaM})$, which binds and activates calcineurin $(\mathrm{CN})$. CN dephosphorylates cytoplasmic NFAT (NFAT-P), thereby exposing a nuclear localization signal. Upon removal of calcium, the sites dephosphorylated by $\mathrm{CN}$ are phosphorylated by glycogen synthase kinase (GSK3), and NFAT is rapidly exported from the nucleus. Thus, persistent activation requires refilling of the IP3-sensitive stores by calcium influx from the external medium. NFAT induction of cytokines and activation of genes involved in morphogenesis is dependent on the concomitant activation of the protein kinase $\mathrm{C}$ (PKC) pathway by DG. In skeletal and cardiac muscle, phosphorylation by a calcium-stimulated kinase and dephosphorylation by $\mathrm{CN}$ of different serines activates the myocyte enhancer binding factor 2 (MEF2). Activation of both MEF2 and NFAT are implicated in muscle differentiation, cardiac hypertrophy and apoptosis. The immunosuppressive drugs FK506 and cyclosporin A (CSA) inhibit the dephosphorylation of NFAT and MEF2.

the importance of a connection between calcium signaling and oxidative stress in his overview of the burgeoning complexities of events that mediate such signaling in living cells.

\section{Calcium-binding proteins and disease}

A number of presentations discussed calcium-signaling dysfunction as an underlying cause of many human diseases. Sitedirected mutagenesis was previously used as a powerful tool by D. MacLennan (Toronto, ON) to demonstrate the role of the SERCA (sarcoplasmic reticulum calcium ATPase) pump in the pathogenesis of malignant hyperthermia (MacLennan, 2000). MacLennan and Carafoli have now extended this approach to both the ryanodine receptor and phospholamban and reported that mutations in these proteins affect calcium homeostasis and may contribute to the pathogenesis of diseases as diverse as the central core disease, familial polymorphic ventricular tachycardia and Brody disease.

In another part of his talk referred to earlier, Olson reviewed the importance of calcineurin in the development of cardiac hypertrophy, an enlargement of the heart arising from most cardiac diseases. Not only can ectopic expression of calcineurin initiate the hypertrophic program, but calcineurin inhibitors such as cyclosporin A can prevent the onset of pressure-induced hypertrophy in rodent models (Olson and Williams, 2000). Crabtree presented evidence that calcineurin plays an important role in the morphogenesis of sensory organs, and defects in the pathways regulated by this phosphatase can lead to congenital cardiac and vascular defects. Angiogenesis defects are observed in mice mutant for the calcineurin regulatory B subunit or NFAT and in mice treated with calcineurin inhibitors, highlighting the relevance of the calcineurin/NFAT-signaling pathway in this important process (Graef et al., 2001). Calmodulin-dependent protein kinases appear to play a role in regulating the immune system, the nervous system and spermatogenesis. Mice that are mutant for one of these enzymes, calcium/calmodulin kinase IV (CaMKIV), display phenotypes that are characteristic of a number of human diseases ranging from osteoporosis to male infertility (A. Means, Durham, NC). Mutations that are linked to the development of Marfan syndrome have been shown to prevent calcium binding to the connective tissue protein, fibrillin (P. Handford, Oxford, UK; Handford, 2000). Overexpression of a centrosomal calmodulin-binding protein called pericentrin is found in numerous cancer cells and can lead to an increased number of centrosomes as well as multipolar spindles, which are, in turn, hallmarks of genomic instability (T. Davis, Seattle, WA; Flory et al., 2002). D. Pietrobon (Padova, Italy) discussed pathological states relating to defects in calcium channels, so-called channnelopathies, of the central nervous system, including spinocerebellar atrophy, familial migraine and delayed cerebellar edema (Pietrobon, 2002). All these conditions are caused by mutations in the voltage-dependent calcium channels. Finally, the dystonic neurobehavioral syndrome may involve malfunction of the neuronal L-type calcium channels (J.Striessnig, Innsbruck, Austria; Platzer et al., 2000). Mouse models null for one of the channel subunits are deaf and exhibit an age-dependent loss of sensory hair cells. Interestingly, this is also a phenotype seen in mice null for CaMKIV, suggesting that multiple calcium-dependent pathways are important for hearing (Means). Thus, the involvement of calcium-signaling pathways in human disease promises to be a fertile avenue for future calcium research.

\section{Reflections}

We continue to be amazed by the large number of cellular and extracellular events that are identified as being subject to calcium regulation. This meeting presented a broad spectrum of current research on calcium and calcium-binding proteins. The posters were numerous and of extremely high quality, which illustrates the fact that the area is attractive to young investigators who work in many countries throughout the world. Certainly, this meeting showed that the future of calcium research is very bright indeed. 


\section{meeting report}

\section{References}

Bayer, K.U., De Koninck, P., Leonard, A.S., Hell, J.W. and Schulman, H. (2001) Interaction with the NMDA receptor locks CaMKII in an active conformation. Nature, 411, 801-805.

Boustany, L.M. and Cyert, M.S. (2002) Calcineurin-dependent regulation of Crz1p nuclear export requires Msn5p and a conserved calcineurin docking site. Genes Dev., 16, 608-619.

Carafoli, E. (2002) Calcium signaling: a tale for all seasons. Proc. Natl Acad. Sci. USA, 99, 1115-1122.

Crabtree, G.R. and Olson, E.N. (2002) NFAT signaling: choreographing the social lives of cells. Cell, 109 (Suppl.), S67-S79.

Drum, C.L., Yan, S.Z., Bard, J., Shen, Y.Q., Lu, D., Soelaiman, S., Grabarek, Z., Bohm, A. and Tang, W.J. (2002) Structural basis for the activation of anthrax adenylyl cyclase exotoxin by calmodulin. Nature, 415, 396-402.

Flory, M.R., Morphew, M., Joseph, J.D., Means, A.R. and Davies, T.D. (2002) Pcp1p, an Spc110p-related calmodulin target at the centrosome of the fission yeast Schizosaccharomyces pombe. Cell Growth Differ., 13, $47-58$.

Graef, I.A., Chen, F., Chen, L., Kuo, A. and Crabtree, G.R. (2001) Signals transduced by $\mathrm{Ca}^{2+} /$ calcineurin and NF-ATc3/c4 pattern the developing vasculature. Cell, 105, 863-875.

Handford, P.A. (2000) Fibrillin-1, a calcium binding protein of extracellular matrix. Biochim. Biophys. Acta, 1498, 84-90.

Hoeflich, K.P. and Ikura, M. (2002) Calmodulin in action: diversity in target recognition and activation mechanisms. Cell, 108, 739-742.

Kovalchuk, Y., Hanse, E., Kafitz, K.W. and Konnerth, A. (2002) Postsynaptic induction of BDNF-mediated long-term potentiation. Science, 295, $1729-1734$.

Lee, S.J. and Montell, C. (2001) Regulation of the rhodopsin protein phosphatase, RDGC, through interaction with calmodulin. Neuron, 32, 1097-1106.

MacLennan, D.H. (2000) $\mathrm{Ca}^{2+}$ signalling and muscle disease. Eur. J. Biochem., 267, 5291-5297.
Moldoveanu, T., Hosfield, C.M., Lim, D., Elce, J.S., Jia, Z. and Davies, P.L. (2002) $\mathrm{A} \mathrm{Ca}^{2+}$ switch aligns the active site of calpain. Cell, 108, 649-660.

Olson, E.N. and Williams, R.S. (2000) Calcineurin signaling and muscle remodeling. Cell, 101, 689-692.

Pietrobon, D. (2002) Calcium channels and channelopathies of the central nervous system. Mol. Neurobiol., 25, 31-50.

Platzer, J., Engel, J., Schrott-Fischer, A., Stephan, K., Bova, S. and Chen, H. (2000) Congenital deafness and sinoatrial node dysfunction in mice lacking class D L-type $\mathrm{Ca}^{2+}$ channels. Cell, 102, 89-97.

Ramulu, P., Kennedy, M., Xiong, W.H., Williams, J., Cowan, M., Blesh, D., Yau, K.W., Hurley, J.B. and Nathans, J. (2001) Normal light response, photoreceptor integrity, and rhodopsin dephosphorylation in mice lacking both protein phosphatases with EF hands (PPEF-1 and PPEF-2). Mol. Cell. Biol., 21, 8605-8614.

Saneyoshi, T., Kume, S., Amaski, Y. and Mikoshiba, K. (2002) The Wnt/ calcium pathway activates NF-AT and promotes ventral cell fate in Xenopus embryos. Nature, 417, 295-299.

Schuchmann, S., Kovacs, R., Kann, O., Heinemann, U. and Buchheim, K. (2001) Monitoring NAD(P)H autofluorescence to assess mitochondrial metabolic functions in rat hippocampal-entorhinal cortex slices. Brain Res. Brain Res. Protoc., 7, 267-276.

Schumacher, M.A., Rivard, A.F., Bachinger, H.P. and Adelman, J.P. (2001) Structure of the gating domain of a $\mathrm{Ca}^{2+}$-activated $\mathrm{K}^{+}$channel complexed with $\mathrm{Ca}^{2+} /$ calmodulin. Nature, 410, 1120-1124.

Sorensen, J.B., Matti, U., Wei, S.H., Nehring, R.B., Voets, T., Ashery, U., Binz, T., Neher, E. and Rettig, J. (2002) The SNARE protein SNAP-25 is linked to fast calcium triggering of exocytosis. Proc. Natl Acad. Sci. USA, 99, 1627-1632.

Teruel, M.N. and Meyer, T. (2002) Parallel single-cell monitoring of receptor-triggered membrane translocation of a calcium-sensing protein module. Science, 295, 1910-1912.

Vandecasteele, G., Szabadkai, G. and Rizzuto, R. (2001) Mitochondrial calcium homeostatis: mechanisms and molecules. IUBMB Life, 52, 213-219.

DOI: 10.1093/embo-reports/kvf182 